\section{The use of mathematical modeling for the construction of a control system for a climate chamber}

\author{
Marian Banaś, Mariusz Filipowicz, Krzysztof Lalik, \\ Sławosz Kleszcz, Szymon Podlasek, Tymoteusz Turlej \\ Mateusz Kozek \\ AGH University of Science and Technology, Poland
}

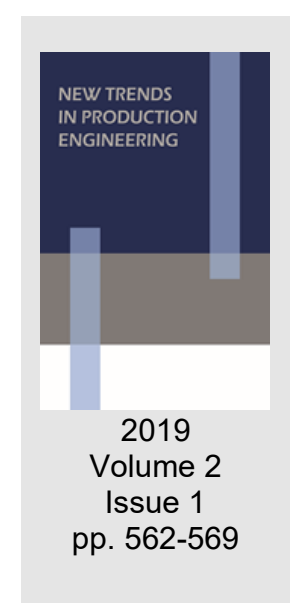

Date of submission to the Editor: 05/2019

Date of acceptance by the Editor: 08/2019

\title{
INTRODUCTION
}

The ever-increasing comfort of human life and increasing awareness of the quality of air forces the need to develop ventilation technology for buildings (Orme, 2001; Calautit et al., 2013; Borowski and Karch, 2015). In addition, the EPBD Directive (Directive 2012/27/EU) introduced by the European Union in 2010 on the energy performance of buildings requires that from 2019 all public buildings, and from 2021 also the remaining ones meet almost zero emission requirements. One of the solutions to achieve this goal, in the case of singlefamily buildings may be the use of mechanical ventilation with heat recovery. Large buildings require solutions with significantly higher efficiency in the form of ventilation units with recuperation. Simplifying the operation of both devices is similar, they are to ensure the best possible air quality and reduce energy consumption by recovering heat from the air (Mijakowski et al., 2010; Vasilyev et al., 2016; Gendebien et al., 2013).

In order to increase the efficiency of this type of devices, it is necessary to study their work in the whole range of possible parameters of the intake air. On the basis of these measurements and the set point for the room, the algorithm implemented in the device controls the heating, cooling and humidifying devices in order to adjust to the setpoints. To carry out tests of the operation of ventilation systems it is necessary to use a specialized research system called a climatic chamber, providing controlled parameters of the air supplied to the tested ventilation device. Particularly important for the testing process of ventilation devices is that the parameters of the intake and exhaust air set in the climatic chamber are constant so that it is possible to determine the operating characteristics in the whole range specified in the relevant standards (PN-EN 13141-7) or assumed by the user.

To achieve this task, it is necessary to use algorithms and control devices, which by means of actuators (pumps, fans, actuators, etc.). One of such solutions is the use of a PID controller that allows effective process control and is widely 
used in industry (PN-EN 13141-7). Due to the industrial nature of the research and further implementation of the solution in the real facility, it was decided to use the PID controller for the purpose of controlling the operating parameters during air treatment for testing ventilation devices.

\section{CHARACTERISTICS OF THE RESEARCH OBJECT}

A climatic chamber is a device that allows to achieve and maintain selected climate parameters, ie temperature, humidity and air flow for any period of time. The key to correct operation is the independence of the set parameters from external atmospheric conditions (the intake is located outside the research room). In this case, the discussed chamber allows the examination of ventilation devices in conditions as close as possible to the real ones. Ventilation system serving the chamber is able to generate $330 \mathrm{~kW}$ of total cooling power and 200 $\mathrm{kW}$ of total heating power, which allows you to control the air temperature in the chamber in the range of -20 to $35^{\circ} \mathrm{C}$. The test chamber (Fig. 1) allows testing devices with a maximum dimension of $2000 \times 2000 \times 3000 \mathrm{~mm}$. (Podlasek et al., 2015). Due to the large surface area of heat exchangers, used in the heating and cooling system of the climate chamber, the system is subject to high thermal inertia which translates into a longer time of temperature stabilization.
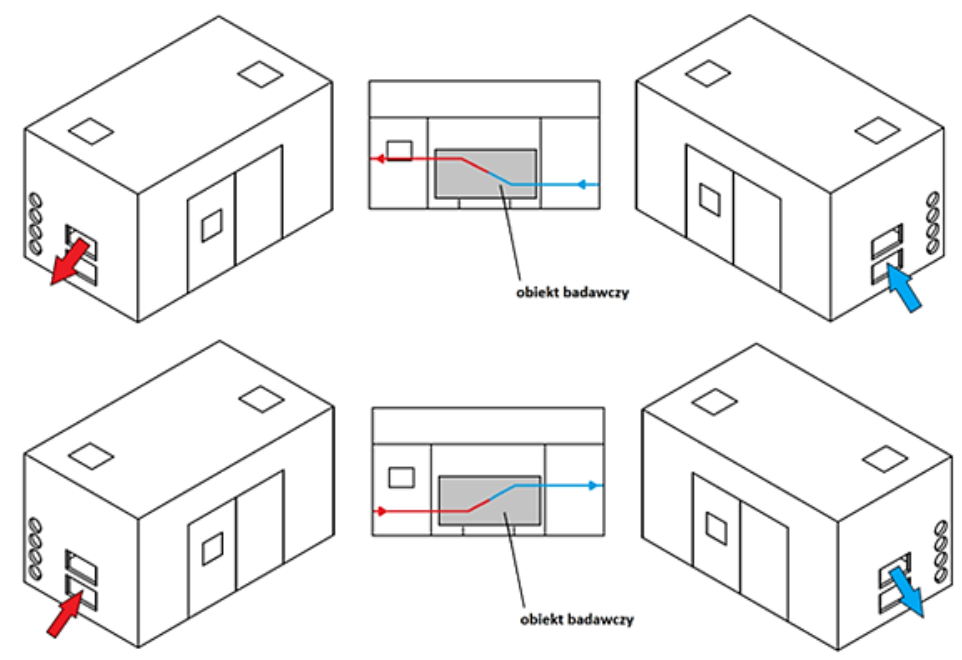

Fig. 1 Schematic diagram of the operation of the climate chamber.

The upper part represents the stream of air drawn, the bottom air drawn from the room

The climatic chamber allows testing of test objects by controlling the air parameters in front of each inlet stub, while the outlet parameters depend on the tested object and the control system implemented in it. The air flows are shown in Figure 1, the direction to the inside of the chamber means the inlet air, the direction to the outside means the exhaust air. The heating and cooling system allows to obtain the following air parameters: derived in the range from $-20^{\circ} \mathrm{C}$ ( $100 \%$ humidity) to $+35^{\circ} \mathrm{C}\left(50 \%\right.$ humidity) at an output of 100 to $7000 \mathrm{~m}^{3} / \mathrm{h}$ and air drawn from the room in range from $+10^{\circ} \mathrm{C}(40-60 \%$ humidity $)$ to $+30^{\circ} \mathrm{C}(60 \%$ humidity) at an output of 100 to $7000 \mathrm{~m}^{3} / \mathrm{h}$. 
From the point of view of control precision, simplifying the idea of cooling in this type of installations, the process consists in directing the circulating medium to the heat exchanger that cools or warms the air flowing through it. Efficiency, and what is also the temperature, can be controlled by changing the speed of rotation of the pumps, or by throttling the flow by means of a control valve. In the case of the test object, a ball valve with an electric actuator as shown in Figure 2 is marked in red. Despite constant control, the actuator's control is not fully precise. This is due to the lack of feedback that determines the actual position of the valve. This phenomenon and the thermal inertia of the exchangers forced the model to adapt to the delays in the system from the moment of task of air parameters to the moment of their real achievement.

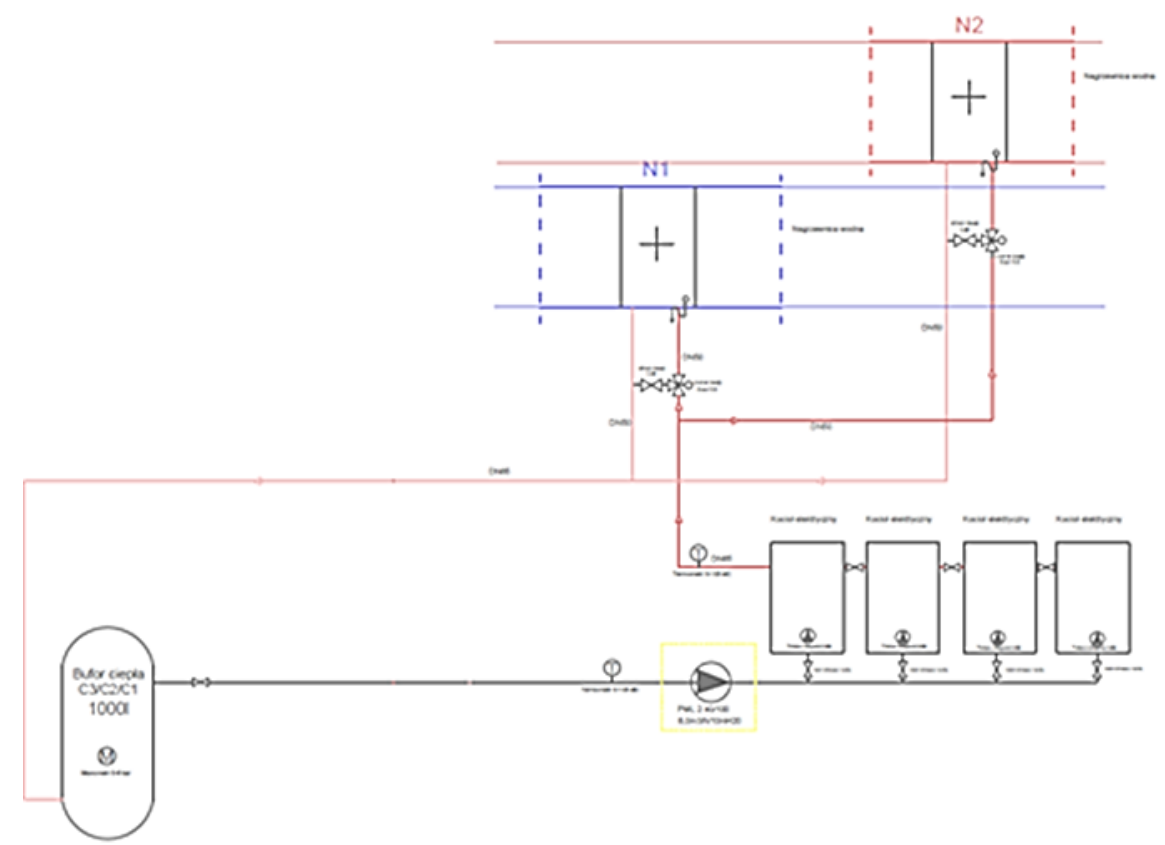

Fig. 2 Hydraulic diagram of the heating part of the climate chamber (space)

For the needs of the research, the operation mode was tested with the parameters: temperature $20^{\circ} \mathrm{C}$ and capacity $2000 \mathrm{~m}^{3} / \mathrm{h}$. Initially, the temperature fluctuations were up to $4^{\circ} \mathrm{C}$, where according to the Polish standard (Mijakowski et al., 2010) it can not differ by more than $1^{\circ} \mathrm{C}$ from the determined value. The previous operation of the climate chamber with standard PID controller settings was characterized by the lack of stable parameters, particularly high fluctuation of the air temperature at the inlet to the ventilation unit. This situation forced the use of specialist settings of the PID regulator, discussed later in the article.

The installation of treatment of air supplied to the tested object consists of two systems: heating visible in Figure 2 and cooling. The first one consists of four gas boilers responsible for supplying warm glycol to heaters (N1 and N2), each with a capacity of $98.3 \mathrm{~kW}$ (Podlasek et al., 2015). They are located on the air ducts in both the exhaust and supply part, on each one. This allows simulations of the work of the tested object both in terms of specific parameters of the air supplied to the room, as well as the extracted one. The cooling system is divided into three parts operating in a cascade. The first stage uses a $67 \mathrm{~kW} \mathrm{CH} 1$ cooler 
that allows to maintain the air temperature in the range from $25^{\circ} \mathrm{C}$ to $6.1^{\circ} \mathrm{C}$. The next level of the cooling system is equipped with two $\mathrm{CH}_{2}$ and $\mathrm{CH}_{3}$ coolers with the capacity of $51.7 \mathrm{~kW}$ allow to achieve a minimum temperature of $-6.8^{\circ} \mathrm{C}$. The redundant system used allows replacing the air flow through exchangers in case of icing of one of them (Seker et al., 2004; Jaszczur et al. 2018). The $\mathrm{CH}_{4}$ cooler with a power of $41.95 \mathrm{~kW}$ is responsible for the last cooling stage and allows the temperature to reach $-21^{\circ} \mathrm{C}$. All radiators are located in the handmade air duct (Podlasek et al., 2015).

\section{NUMERICAL SIMULATION MODEL}

The purpose of the work was to improve the original structure of the control system with block mixer valves. This structure caused incorrect operation of the system. The most unfavorable phenomenon in the regulation was obtaining a high over-regulation value, which caused that the air in the exchanger block reached an unstable temperature significantly different from the set one (Fig. 3). The main purpose of the work was to eliminate this phenomenon and achieve high quality control by eliminating the error from the temperature value exchanger, significant reduction of over-regulation and improvement of regulation time.
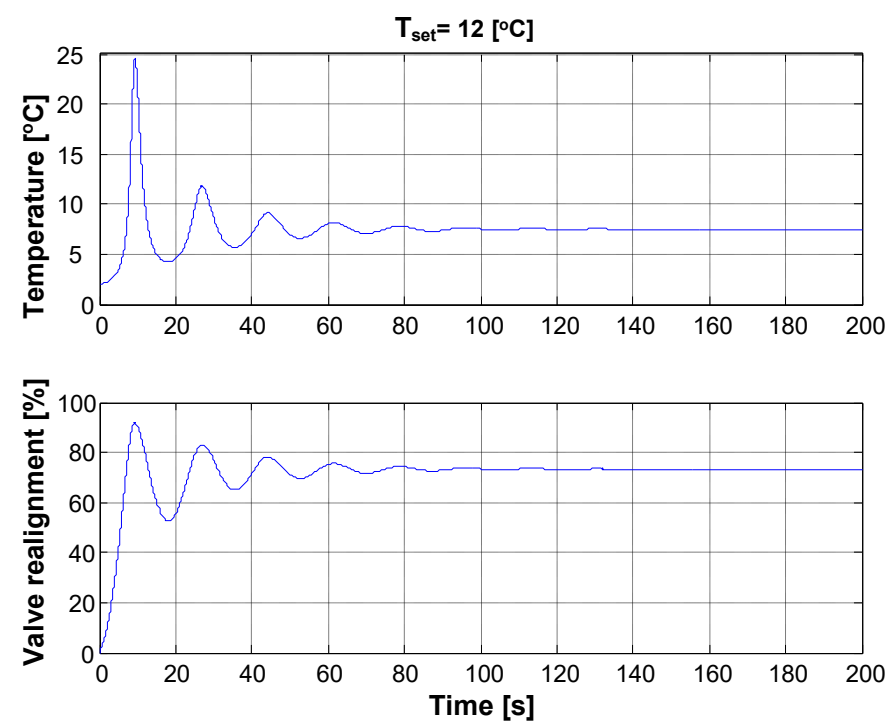

Fig. 3 Characteristics of the system with insufficient control quality (pre-arrangement)

The estimation of PID controller parameters usually takes place in a heuristic manner. On the one hand, this methodology is the simplest in implementation, but it does not even guarantee suboptimal results. In addition, in the case of objects with high inertia, which are most often objects in which the process variable is regulated temperature, this methodology is very time-consuming. This is due to the need to bring the system into its original state every time. Therefore, the test of each set of PID controller parameter settings requires that the system be brought to the same starting temperature. This article proposes a different method for the selection of optimal PID controller settings. 
The methodology of the proposed solution is based on a strictly defined procedure. In the research studies, the following steps were carried out:

- Identification experiment. The real system has been activated by a step force signal. In the open control system, a constant value of the valve cover was applied. Next, the change of the process variable (temperature) after the system was examined. Both the extortion signal and the system response have been registered. The choice of extortion was not accidental. In control theory, step excitation represents the method of energy storage by the system. This allows you to minimize the integral adjustment indicator of the square type of the control error. This indicator is directly interpreted as energy lost in the regulation process.

- Identification tool. On the basis of the measurement, an attempt was made to obtain a mathematical representation of the control object in the form of an operator transfer function. Such a model allowed for later optimization of the regulator's settings. Parametric models were used for identification. These models are by far the most important family of models representing stationary time series. If the time series does not show excessive deviations from stationarity and its autocorrelation function (autocorrelation) disappears quite quickly, it is usually considered these models as suitable for data representation. Based on these models, a numerical representation of the object was obtained.

- PID controller optimization tool. Thanks to the model of the system obtained in the previous step, it was possible to create a structure that allowed to optimize the PID controller settings. A gradient goal minimization algorithm was used for this purpose. In gradient methods, the search direction is created in each iteration on an ongoing basis based on information about the gradient of the objective function at the point reached. This algorithm allowed to obtain the optimal control trajectory

- Verification of received settings on the real object.

The transmittance of the valve model has been assumed to be the first order inertial object defined by equation 1. The transmittance of such a object processes the input signal without restrictions, while the actual valve can only reach the cover value in the range from fully closed to fully open. Hence, the saturation model was imposed on the numerical model, which was to reproduce the actual operation of the valve.

where:

$$
G_{O}(s)=\frac{K}{T s+1}
$$

Go - valve transfer function,

$\mathrm{K}$ - amplification of the system,

$\mathrm{T}_{\mathrm{s}}$ - time constant of the system.

It is possible to assume that the input and output signals for a certain time interval are stationary. Hence, it allows to identify the transmittance of the object using a parametric model (Equation 2). They use operator's record of dynamics of the system in the form of transmittance. For the adopted description of the 
system in the form of input and output tracks and the influence of disturbances on the output path. Hence, for a parametric polynomial model, the disturbance is described as the object brought to the output.

$$
y_{i}=G\left(z^{-1}\right) x_{i}+H\left(z^{-1}\right) e_{i}
$$

where:

$y$ - output signal,

$\mathrm{x}$ - input signal,

e - interference signal

$\mathrm{G}$ - transmittance of the object,

$\mathrm{K}$ - amplification of the system,

$\mathrm{H}$ - transmittance of interference filter, on the input of which is white noise.

The identification of the $\mathrm{G}\left(z^{-1}\right)$ object model and the $\mathrm{H}\left(z^{-1}\right)$ interference model) is possible directly or indirectly. The indirect method requires knowledge of the preset signal that was available as a result of the identification experiment. The direct method has been described in (Li et al., 2006).

The identification model was based on the mathematical algorithm of parametric models and allowed to obtain a discrete transmittance of the real system. After transformation into a continuous domain, it takes the form of dependence (3).

$$
G(s)=\frac{0.7728 s^{3}-1.533 s^{2}-0.02539 s-3.518 e-007}{s^{3}+2.017 s^{2}+0.03316 s+1.313 e-008}
$$

The obtained model was used to determine the optimal regulator settings.

Based on the numerical simulations, the PID controller parameters were obtained, which were then introduced into the actual controller and verified on the real object. The operation of the tuned system is shown in Figure 4. The tests carried out confirm the correct operation of the system. The error of the controlled value has been eliminated, and over-regulation has been significantly reduced.
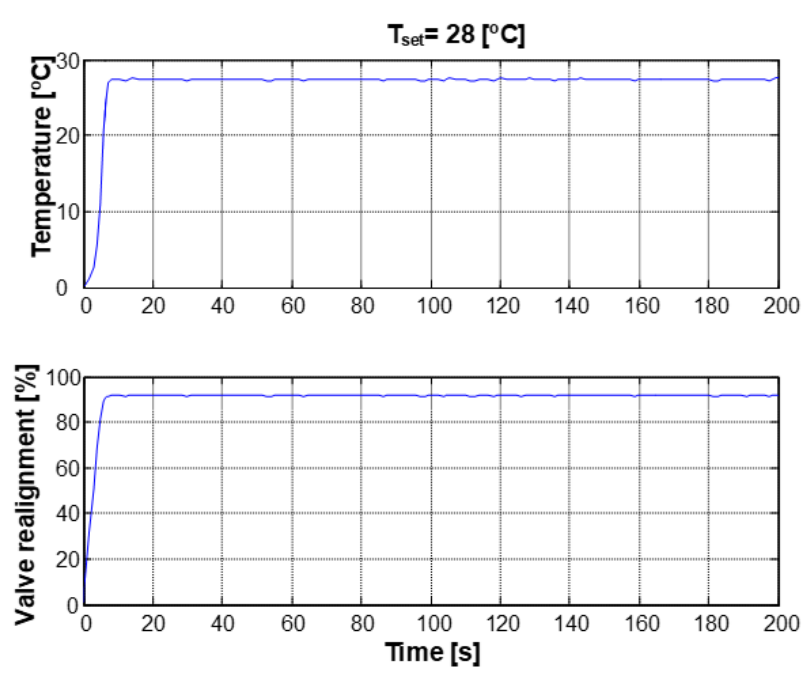

Fig. 4 Operation of the system during operation determined with selected controller settings

The PI controller structure was assumed in the model. The differentiating part was abandoned due to two aspects. The time constant of the object was so large 
that the operation of the differentiating part did not significantly affect the controller's operation in the transient state. In turn, too high a value of the derivative would cause that if in a steady state there would be significant disturbances, then the derivative would lead to oscillation of the controlled value.

\section{CONCLUSION}

In industry, optimally tuned PID controllers are very rare. This is due to the fact that the classical methods of tuning the regulator are either too time-consuming or too complicated. Obviously, the wrong selection of these settings directly leads to large energy losses. This article provides a quick method to overcome these obstacles. The presented method relies on a certain procedure and the use of engineering tools built into the Matlab/Simulink package.

The results presented in the article show the advantages of the presented method over heuristic methods. This is true both in terms of time consumption and the quality of the achieved regulation. Our research should focus on the development of a specialized function block that could be implemented directly in the microprocessor control unit. It will consist of an identification module, a numerical simulation module and an optimizing module. It should be able to choose an optimization tool, because, especially for non-stationary facilities, the regression methods may not guarantee optimal results.

\section{ACKNOWLEDGEMENTS}

Work carried out under the rector's grant number 80/GRANT/2019

\section{REFERENCES}

Borowski M., Karch M. (2015), Chłodnictwo \& Klimatyzacja 4, 65

Calautit J.K., Hughes B.R., Chaudhry H.N., Ghani S.A., (2013) Applied Energy 112, 576

Directive 2012/27/EU of the European Parliament and of the Council of 25 Oct. (2012) Gendebien S., Bertagnolio S., Lemort V. (2013), Energy and Buildings 62, 176

Jaszczur M., Kleszcz S., Borowski M. (2018), Analysis of the anti-icing system used in air handling units with a counterflow heat exchanger

Li Y., Ang K.H., Chong G.C.Y. (2006), IEEE Control Systems Magazine, 26, 1

Mijakowski M., Sowa J., Narowski P. (2010), Journal of Civil Engineering 4, 107

Orme M., Energy Build (2001), 33, 199

Podlasek S., Lalik K., Filipowicz M., Sornek K., Kupski R., Raś A. (2015), Mathematical modeling of control system for the experimental steam generator, EFM, Praga

Polish regulation PN-EN 13141-7

Seker D., Kartas H., Egrican N. (2004), International Journal of Refrigeration 27, 367

Vasilyev G.P., Tabunshchikov A., Brodach M.M., Leskov V.A., Mitrofanova N.V., Timofeev N.A., Gornov V.F., Esaulov G.V. (2016), Energy and Buildings 112, 96 


\begin{abstract}
.
Due to the introduced political instruments, as well as the increase in awareness and standard of living, mechanical ventilation is becoming more and more popular in Poland. The growing market and standards force the manufacturers of air handling units to constantly improve the quality of their products. In order to verify the operation of these devices, it is necessary in a specially adapted for this object called the climate chamber. Due to the strict regulations regarding the working conditions of the said facilities, it is necessary to apply the processes of advanced control systems in the process of regulation. These processes are aimed at establishing stable parameters of air supplied to the tested objects, ventilation and airconditioning units such as: temperature, humidity, flux. Due to the need for precise control and operation of the installation in industrial conditions, it was decided to use the PID controller. The article deals with the optimization of the heating and cooling system, because the temperature parameter was a problematic element in the proper operation of the climate chamber. Both the heating and cooling systems have been described and executive elements have been emphasized, thanks to which it was possible to control the flow of circulating factors. The procedure of selection and implementation of the regulator's settings and its influence on the operation of the climate chamber was also analyzed.
\end{abstract}

Keywords: ventilation, recuperation, climatic chamber, PID controller, optimization 